\title{
Structural Characterization of the Activated Leptin Receptor Complex
}

\author{
L. Mancour ${ }^{1,2}$, J. Schilling ${ }^{1}$ and G. Skiniotis ${ }^{1,2}$ \\ ${ }^{1}$ Life Sciences Institute, University of Michigan, 210 Washtenaw Ave., Ann Arbor, MI 48109-2216 \\ 2 Department of Biological Chemistry, University of Michigan, 210 Washtenaw Ave., Ann Arbor, \\ MI 48109-2216
}

Leptin is a hormone, secreted from adipocytes in levels proportional to body fat, and binds to the leptin receptor (L-R) in the hypothalamus, thereby regulating body energy homeostasis. Leptin signaling reduces food intake while it increases energy expenditure, and mutations in either the hormone or its receptor result in marked obesity in both mice and humans. Beyond this role, leptin action is also heavily implicated in hyperinsulinaemia and type 2 diabetes, tumorigenesis, angiogenesis, bone formation, sexual maturation, immunity and wound healing.

The L-R is a transmembrane protein with an extracellular domain organization that is similar to other members of the class I cytokine receptor family. The ectodomain is characterized by two cytokine receptor homology region (CRH1 and CRH2) separated by an Ig-like domain, and also includes two fibronectin III (FNIII) membrane proximal domains. While the CRH2 module serves as the primary ligand binding site [1], the Ig-like and FNIII domains have also been implicated in complex formation and receptor activation. Binding of the cytokine to the extracellular portion of the leptin receptor is thought to induce a conformational change that promotes transphosphorylation of constitutively bound tyrosine janus kinase 2 (JAK2) on the intracellular site of the receptor.

The mechanism of activation of L-R as well as the exact stoichiometry of the complex is controversial $[2,3]$. Additionally, the relative large molecular size $(\sim 170 \mathrm{kDa})$ and flexibility of the receptor make its structural determination with techniques like NMR and x-ray chrystallography exceedingly challenging. We employ single-particle electron microscopy to structurally characterize the extracellular portion of the receptor alone and in complex with leptin.

Our results show that the extracellular portion of L-R is a monomer in solution, but forms a dimer after incubation with leptin in a 2:2 stoichiometric ratio. 2D EM averages and modeling of the L/L-R complex suggest that leptin employs conserved epitope II to engage the CRH2 of one L-R, while epitope III engages the Ig-like domain of a second L-R. In addition, the FNIII domains of the 2 L-R chains appear to interact at their C-terminal tips, by a homotypic interaction not observed in the nonliganded receptor. We postulate that the close proximity and resulting orientation of the membrane proximal FNIII domains is promoting JAK2 transphosphorylation on the intracellular side of the receptor.

References

[1] H. Iserentant et al., Journal of Cell Science, 118, (2005), 2519

[2] F. Peelman et al., JBC, 281, (2006), 15496

[3] C. Couturier and R.Jockers, $J B C, 278,(2003), 26604$

[4] The project described was supported by grant number GM007767 from NIGM and its contents are solely the responsibility of the authors and do not necessarily represent the official views of NIGMS 